Canad. Math. Bull. Vol. 21 (4), 1978

\title{
ON THE POWER MAP AND RING COMMUTATIVITY
}

\author{
BY \\ HOWARD E. BELL*
}

Let $R$ denote an associative ring with 1 , let $n$ be a positive integer, and let $k=1,2$, or 3 . The ring $R$ will be called an $(n, k)$-ring if it satisfies the identities

$$
(x y)^{m}=x^{m} y^{m}
$$

for all integers $m$ with $n \leq m \leq n+k-1$. It was shown years ago by Herstein (See [2], [9], and [10]) that for $n>1$, any $(n, 1)$-ring must have nil commutator ideal $C(R)$. Later Luh [12] proved that primary $(n, 3)$-rings must in fact be commutative, and Ligh and Richoux [11] recently showed that all $(n, 3)$-rings are commutative. Luh gave an example showing that $(n, 2)$-rings need not be commutative; Awtar [1] and Harmanci [5], using rather complicated combinatorial arguments, established commutativity of $(n, 1)$-rings and $(n, 2)$-rings in which the additive group $R^{+}$is $p$-torsion-free for all primes $p \leq n$.

The first theorem of this note improves the latter results for $(n, 2)$-rings by relaxing the torsion restrictions and, incidentally, provides a much simpler proof of Harmanci's result; and the next two theorems provide different kinds of commutativity conditions for $(n, 2)$-rings. The remainder of the paper deals with commutativity conditions for rings which are either radical over their center or satisfy the identity $x^{n} y-y x^{n}=x y^{n}-y^{n} x$ for some $n>1$.

Throughout the paper, we shall denote the commutator $x y-y x$ by $[x, y]$, the center of $R$ by $Z$, and the commutator ideal by $C(R)$.

\section{Commutativity theorems for $(n, 2)$-rings}

THEOREM 1. Let $n$ be any positive integer. If $R$ is any $(n, 2)$-ring for which $R^{+}$ is $n$-torsion-free, then $R$ is commutative.

Proof. Following Ligh and Richoux, we note that $x^{n+1} y^{n+1}=(x y)^{n+1}=$ (xy) $x^{n} y^{n}=x^{n} y^{n} x y$; hence

$$
x\left[x^{n}, y\right] y^{n}=0 \quad \text { and } \quad x^{n}\left[x, y^{n}\right] y=0 \text { for all } x, y \in R .
$$

Replacing $y$ by $y+1$ in the first equation of (1) and right-multiplying by $y^{n-1}$, we see that $x\left[x^{n}, y\right] y^{n-1}=0$; a similar argument applied to the second equation of (1) gives $x^{n-1}\left[x, y^{n}\right] y=0$. Repetition of this argument, together with an

Received by the editors October 20, 1977 and, in revised form, February 3, 1978.

* Supported by the National Research Council of Canada, Grant No. A3961. 
interchange of $x$ and $y$ in the computations involving the second equation of (1), eventually gives

$$
x\left[x^{n}, y\right]=\left[x^{n}, y\right] x=0 \quad \text { for all } \quad x, y \in R .
$$

It follows at once that $x^{n} \in Z$ for all invertible elements $x$; and since $u$ nilpotent implies $1+u$ is invertible, we have

$$
1+n u+v \in Z \text { for all nilpotent elements } u,
$$

where $v=\left(\begin{array}{l}n \\ 2\end{array}\right) u^{2}+\left(\begin{array}{l}n \\ 3\end{array}\right) u^{3}+\cdots$. Now (3) implies that for $u$ with $u^{2}=0, n u \in Z$ and hence $u \in Z$. Proceeding inductively on the assumption that $u^{j}=0$ with $j<k$ implies $u \in Z$, we consider $u$ with $u^{k}=0$ and note that the corresponding $v$ satisfies $v^{k-1}=0$, so that (3) again forces $n u \in Z$ and hence $u \in Z$. Thus, all nilpotent elements are central.

Now $R$ is an $(m, 1)$-ring for at least one $m>1$, so Herstein's result guarantees that commutators are nilpotent, hence central. It is well known, and easily provable by induction, that for rings with central commutators,

$$
\left[x^{m}, y\right]=m x^{m-1}[x, y] \text { for all integers } m \geq 1 \text { and all } x, y \in R .
$$

Applying this in the case $m=n$ and recalling (2), we get

$$
0=x\left[x^{n}, y\right]=n x^{n}[x, y]
$$

we now use the absence of $n$-torsion to get $x^{n}[x, y]=0$ for all $x, y \in R$. Finally, replacing $x$ by $x+1$ and proceeding as at the beginning of the proof, we get $[x, y]=0$ for all $x, y \in R$.

THEOREM 2. Let $n$ and $m$ be relatively prime positive integers. Then any ring $R$ which is both an $(n, 2)$-ring and an $(m, 2)$-ring is commutative.

Proof. The proof above needs only trivial modification. Let $p$ and $q$ be integers such that $1=p m+q n$. At each stage of the inductive argument involving nilpotent elements, the $n$ and $m$ versions of (3) show that $n u$ and $m u \in Z$; thus $u=p m u+q n u \in Z$. Similarly, at the end of the proof we get $n x^{n}[x, y]=m x^{m}[x, y]=0$, and hence $m x^{t}[x, y]=n x^{t}[x, y]=0$, where $t$ is the larger of $m$ and $n$; thus, invoking the relative-primeness of $m$ and $n$ shows $x^{t}[x, y]=0$ and hence $[x, y]=0$ for all $x, y \in R$.

It was shown in [4] that for $n>1$, if $R$ is a ring generated by its $n$th powers and if the map $x \rightarrow x^{n}$ is an additive endomorphism, then $R$ is commutative. It is natural to inquire whether a similar result holds if the $n$ th-power map is an endomorphism of the multiplicative semigroup-i.e. if $R$ is an $(n, 1)$-ring. Luh's example of a non-commutative $(3,2)$-ring shows that this is not the case, for it is a $(4,1)$-ring generated by its fourth powers; however, for $(n, 2)$-rings, a result of this kind does hold. 
THEOREM 3. Let $n$ be any positive integer. Then any $(n, 2)-$ ring which is generated as a ring by either its $n^{2}$-powers or its $n(n+1)$-powers is commutative.

Proof. Consider first the case of $R$ generated by its $n^{2}$-powers. Replacing $y$ by $y^{n}$ in (2), we get $x^{n}\left(x^{n} y^{n}-y^{n} x^{n}\right)=\left(x^{n} y^{n}-y^{n} x^{n}\right) x^{n}=0$; thus, for arbitrary $n$ th-powers $a$ and $b$ we have $a^{2} b=a b a=b a^{2}$. It follows at once that $a^{n} b=$ $b a^{n}$, so that $n^{2}$-powers commute and $R$ is commutative.

Now suppose $R$ is generated by its $n(n+1)$-powers. By applying (2), we get

$$
\left[x^{n+1}, y\right]=x\left[x^{n}, y\right]+[x, y] x^{n}=[x, y] x^{n}
$$

replacing $x$ by $x^{n}$ and again using (2) gives $\left[x^{n(n+1)}, y\right]=0$ so that $R$ is commutative.

2. Further commutativity theorems. The use of equation (4) in the proofs of Theorems 1 and 2 depends on the fact that $(n, 2)$-rings have nil commutator ideal. Among other classes of rings in which $C(R)$ is known to be nil are (i) rings radical over their center-i.e. rings in which some power of each element is central [7]; (ii) rings satisfying the identity $\left[x^{n}, y\right]=\left[x, y^{n}\right]$ for some $n>1$ [4]. (The latter class includes the rings for which the $n$ th-power map is an additive endomorphism.) The remaining theorems state sufficient conditions for full commutativity of certain of these rings. The proof of Theorem 4 is omitted, since it is very similar to those of Theorems 1 and 2 .

THEOREM 4. Let $R$ be a ring with 1 which satisfies one of the following conditions:

(A) $R$ is radical over its center and $R^{+}$is torsion-free;

(B) For a fixed integer $n>1, R^{+}$is $n$-torsion-free; and for each $x \in R$, there exists an integer $k=k(x)$ such that $x^{n^{k}} \in Z$;

(C) For each $x \in R$, there exists a pair $p$, q of relatively prime positive integers for which $x^{p} \in Z$ and $x^{q} \in Z$.

Then $R$ is commutative.

THEOREM 5. Let $R$ be a ring with 1 and $n>1$ a fixed positive integer. If $R^{+}$is $n$-torsion-free and $R$ satisfies the identity

$$
x^{n} y-y x^{n}=x y^{n}-y^{n} x,
$$

then $R$ is commutative.

Proof. As in our previous proofs, we show by induction that nilpotent elements are central. Note first that if $u$ is nilpotent and $y$ is arbitrary,

$$
\left[u, y^{n}\right]=\left[u^{n}, y\right]
$$

and

$$
\left[1+u, y^{n}\right]=\left[1+n u+\left(\begin{array}{c}
n \\
2
\end{array}\right) u^{2}+\cdots u^{n}, y\right]
$$


Thus, if $u^{2}=0, u$ commutes with all $n$ th-powers by (6); and (7) then shows that $[n u, y]=0$ and hence $[u, y]=0$. Now suppose that if $u^{j}=0$ with $j<k$, then $u$ is central; consider $u$ with $u^{k}=0$. Then $u^{2}, u^{3}, \ldots, u^{n}$ are all central, so (6) shows $u$ commutes with $n$ th-powers, and (7) then yields the result that $n u \in Z$, hence $u \in Z$.

Since we now know that $C(R) \subseteq Z$, we shall routinely use equation (4) without explicit mention. In particular, the following properties of $R$, and hence of any homomorphic image of $R$, follow as in [4]:

$$
\begin{aligned}
& n\left[x^{n}, y\right]\left(x^{n(n-1)}-x^{n-1}\right)=0 \text { for all } x, y \in R \\
& x^{q} \in Z \text { for all } x \in R, \text { where } q=n\left(2^{n}-2\right) .
\end{aligned}
$$

Represent $R$ as a subdirect sum of a family $\left\{R_{\alpha}\right\}$ of subdirectiy irreducible rings which are homomorphic images of $R$. Clearly, each $R_{\alpha}$ has 1 , satisfies $(\dagger)$, has central commutator ideal, and satisfies (8) and (9); however, we cannot assume that $\boldsymbol{R}_{\alpha}^{+}$is $n$-torsion-free. It is our immediate aim to show that each $\boldsymbol{R}_{\alpha}$ satisfies the identity $\left[x^{m}-x, y^{n^{2}}\right]=0$, where $m=q(n-1)+1, q$ being as in (9) above.

Let $S$ be the heart of $R_{\alpha}$ - that is, the intersection of all non-zero ideals; and note that if $d$ is a central zero divisor, then $S d=0$, since the annihilator of $d$ is a two-sided ideal and must therefore contain $S$. Now let $a$ be an arbitrary zero divisor in $R_{\alpha}$. (There is no distinction between left and right zero divisors since commutators are central.) For arbitrary $y \in R$, we get from (8) the result that $n\left[a^{n}, y\right]\left(a^{n(n-1)}-a^{n-1}\right)=0$. Multiplying this by appropriate powers of $a^{(n-1)^{2}}$ and subtracting, we see that

$$
n\left[a^{n}, y\right] a^{n-1} f=0,
$$

where $f=1-a^{(n-1)^{2} a}$. Let $T=\left\{x \in R_{\alpha} \mid x y f=0\right.$ for all $\left.y \in R_{\alpha}\right\}$; note that $T$ is a two-sided ideal and that, in view of (10) and the centrality of $C(R)$, $n\left[a^{n}, y\right] a^{n-1} \in T$ for all $y \in R_{\alpha}$. If $T$ is non-trivial, then $S \subseteq T$; and since $S$ annihilates central zero divisors, for each non-zero $s \in S$ we get $0=s f=$ $s-s\left(a^{q}\right)^{(n-1)^{2}}=s-\mathrm{a}$ contradiction. Thus, $T=\{0\}$ and $n\left[a^{n}, y\right] a^{n-1}=0$ for all $y \in R_{\alpha}$. It follows that

$$
\left[a, y^{n^{2}}\right]=\left[a^{n^{2}}, y\right]=\left[\left(a^{n}\right)^{n}, y\right]=n\left[a^{n}, y\right] a^{n(n-1)}=0
$$

for all $y \in R_{\alpha}$ and all zero divisors $a \in R_{\alpha}$.

Suppose now that there exists some $b \in R_{\alpha}$ which does not commute with $n^{2}$-powers. Then $b$ is not a zero divisor, and there exists $r \in R_{\alpha}$ for which $\left[b, r^{n}\right] \neq 0$. For arbitrary $z \in Z$, repiacing $x$ by $z x$ in (†) yields $\left(z^{n}-z\right)\left[x, y^{n}\right]=0$ for all $x, y \in R$; in particular, $\left(b^{n q}-b^{q}\right)\left[b, r^{n}\right]=0$, so that $b^{n q}-b^{q}$, and hence also $b^{q(n-1)+1}-b$ is a zero divisor. Thus, if $m=q(n-1)+1$, it follows from (11) that $\left[x^{m}-x, y^{n^{2}}\right]=0$ for all $x, y \in R_{\alpha}$. 
It is now clear that our original ring $R$ also satisfies the identity

$$
\left[x^{m}-x, y^{n^{2}}\right]=0 \text {. }
$$

Moreover, since $R^{+}$is $n$-torsion-free, $\left[w, y^{n^{2}}\right]=0=n^{2} y^{n^{2-1}}[w, y]$ for all $y \in R$ implies $y^{n^{2}-1}[w, y]=0$ for all $y \in R$; employing the device of replacing $y$ by $y+1$ as in our earlier proofs, we get the result that $w \in Z$. From (12) it follows that $x^{m}-x \in Z$ for all $x \in R$; by a well-known theorem of Herstein (See [3] or [6]), this forces $R$ to be commutative.

Harmanci showed in [5] that if $n>1$ and $R$ is a ring with 1 which satisfies the identities $\left[x^{n}, y\right]=\left[x, y^{n}\right]$ and $\left[x^{n+1}, y\right]=\left[x, y^{n+1}\right]$, then $R$ must be commutative. The methods of our last proof yield the following generalization of that result.

THEOREM 6. Let $m$ and $n$ be relatively prime integers greater than 1. If $R$ is any ring with 1 satisfying both the identities $\left[x^{m}, y\right]=\left[x, y^{m}\right]$ and $\left[x^{n}, y\right]=$ $\left[x, y^{n}\right]$, then $R$ is commutative.

Proof. The beginning of the proof of Theorem 5 can easily be modified to show that nilpotent elements are central under the present hypotheses. The argument for subdirectly irreducible rings can then be carried out for both $m$ and $n$, yielding integers $j, k>1$ such that $R$ satisfies the identities $\left[x^{j}-x, y^{m^{2}}\right]=$ 0 and $\left[x^{k}-x, y^{n^{2}}\right]=0$. Letting $p(x)=\left(x^{j}-x\right)^{k}-\left(x^{j}-x\right)$, we see that $0=$ $\left[p(x), y^{m^{2}}\right]=m^{2} y^{m^{2-1}}[p(x), y]$ and $0=\left[p(x), y^{n^{2}}\right]=n^{2} y^{n^{2}-1}[p(x), y]$ for all $x, y \in$ $\boldsymbol{R}$. The relative primeness of $m$ and $n$ yields $y^{t}[p(x), y]=0$ for all $x, y \in R$, where $t=\max \left\{m^{2}-1, n^{2}-1\right\}$; and it follows as usual that $p(x)$ is central. Since $p(x)$ has form $x-x^{2} q(x)$ with $q$ having integer coefficients, the theorem of [8] shows that $R$ is commutative.

REMARK. In Theorem 5, the restriction on $n$-torsion is essential. To see this, begin with Harmanci's Example 1 [5, p. 29] and use the Dorroh construction (with the ring of integers mod.2) to obtain a ring $R$ with 1 . This ring $R$ is non-commutative and satisfies the identity $\left[x^{2}, y\right]=\left[x, y^{2}\right]$.

\section{REFERENCES}

1. R. Awtar, On the commutativity of non-associative rings, Publ. Math. Debrecen 22 (1975), 177-188.

2. H. E. Bell, On a commutativity theorem of Herstein, Arch. Math. 21 (1970), 265-267.

3. _- Certain near-rings are rings, J. London Math. Soc. (2) 4 (1971), 264-270.

4. —_, On some commutativity theorems of Herstein, Arch. Math. 24 (1973), 34-38.

5. A. Harmanci, Two elementary commutativity theorems for rings, Acta. Math. Acad. Sci. Hungar. 29 (1977), 23-29.

6. I. N. Herstein, A generalization of a theorem of Jacobson, Amer. J. Math. 73 (1951), 756-762.

7. - A theorem on rings, Canadian J. Math. 5 (1953), 238-241.

8. —- The structure of a certain class of rings, Amer. J. Math. 75 (1953), 864-871.

9. —- Power maps in rings, Michigan Math. J. 8 (1961), 29-32.

10. —, A remark on rings and algebras, Michigan Math. J. 10 (1963), 269-272. 
11. S. Ligh and A. Richoux, A commutativity theorem for rings, Bull. Austral. Math. Soc. 16 (1977), 75-77.

12. J. Luh, A commutativity theorem for primary rings, Acta. Math. Acad. Sci. Hungar. 22 (1971), 211-213.

Department of Mathematics

BROCK UNTVERSTTY

St. Catharines, Ontario, Canada

L2S 3A1 